\title{
S-19-3 Theoretical Basis for Antioxidant Action in Disease Prevention
}

\author{
A.T.DiploCK
}

Division of Biochemistry, United Medical and Dental Schools, (University of London) Guy's Hospital, LONDON, SE1 9RT, United Kingdom.

\section{INTRODUCTION}

The understanding of the nature of free radical-driven chemical reactions among biological macromolecules in living mammalian cells, and of their control by a complex range of interacting nutrients, and nutrientderived factors, is a new area of biochemistry that lies at the heart of the explanation of the aetiology of a number of diseases including importantly some forms of cancer and cardio-vascular disease [1]. The four-electron reduction of molecular dioxygen may be considered to take place in a series of single electron stages. The first stage involves the formation of the superoxide anion radical $\mathrm{O}_{2}{ }^{-}$and in the second stage there is a further reduction of $\mathrm{O}_{2}$ - to a peroxyl anion $\left(\mathrm{O}_{2}^{--}\right)$by the addition of a further single electron to the molecule. Neither of these species is particularly reactive towards biological macromolecules and it is the further metabolism of these oxygen metabolites that poses a threat to intact mammalian cells. The uncontrolled generation of oxygen species, and the absence of control of the proliferation of secondary metabolites, are the processses that are believed to give rise to a range of different diseases. Thus, a major current hypothesis [2] on the aetiology of cardiovascular disease depends on the concept that peroxidized lipid in the serum low density lipoprotein fraction is a determining factor in atherogenesis; furthermore, there is little doubt that free radicals are involved in the aetiology of some forms of cancer [3], and free radicals have been implicated in a range of other diseases notable among which is cataract [4].

\section{ANTIOXIDANT NUTRIENTS}

The control of the oxidative processes that are believed ultimately to lead to disease depends upon an intimate interplay of a number of essential nutrients. These include the trace minerals $\mathrm{Mn}, \mathrm{Cu}, \mathrm{Zn}$ and $\mathrm{Se}$, vitamins $\mathrm{E}$ and $C$ and probably also involves beta carotene which, as distinct from its provitamin A role, has not usually been thought to have a nutritional role in its own right. The proliferation of formation of the hydroxyl radical, which, because of its very high reaction rate constants, is the primary candidate for initiating oxygen radical damage, is driven by an iron catalysed process thus:

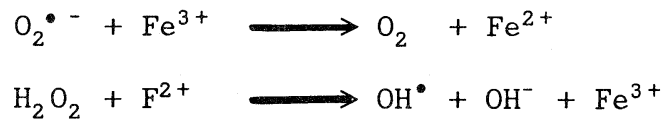

The hydroxyl radical so generated, if it occurs in biological systems, will react very rapidly with biological macromolecules in its immediate vicinity. This process, which may involve DNA, proteins (enzymes) and, particularly, membrane polyunsaturated phospholipids, is thought to lead to the alterations in cellular architecture that are seen as the pathogenic process of disease. 
It is clear, in considering the foregoing equations, that the process may theoretically be controlled in two ways: by limiting the amount and availability of iron and/or by removing the $\mathrm{O}_{2}{ }^{-}$and $\mathrm{H}_{2} \mathrm{O}_{2}$ substrates from the system.

Living mammalian cells possess enzymes that are capable of catalyzing the removal of $\mathrm{O}_{2}^{--}$and $\mathrm{H}_{2} \mathrm{O}_{2}$. Within the mitochondrial compartment and within the cytoplasm, a superoxide dismutase enzyme (SOD) catalyses the following reaction:

$2 \mathrm{O}_{2}^{\bullet-}+2 \mathrm{H}^{+} \longrightarrow \mathrm{H}_{2} \mathrm{O}_{2}+\mathrm{O}_{2}$

The mitochondrial enzyme contains $M n$, and the cytoplasmic enzyme contains $\mathrm{Cu}$ and $\mathrm{Zn}$, and the efficient catalytic activity of these enzymes depends on an adequate nutritional supply of these minerals. Hydrogen peroxide ${ }_{i} \mathrm{~s}$ removed by two enzymes: catalase, catalyzes the disproportionation of $\mathrm{H}_{2} \mathrm{O}_{2}$ thus:

$2 \mathrm{H}_{2} \mathrm{O}_{2} \longrightarrow \mathrm{H}_{2} \mathrm{O}_{2}+\mathrm{O}_{2}$

Perhaps more importantly, glutathione peroxidase, a selenoenzyme that depends on the nutritional availability of Se, catalyze the reduction by glutathione of $\mathrm{H}_{2} \mathrm{O}_{2}$ thus:

$\mathrm{H}_{2} \mathrm{O}_{2}+2 \mathrm{GSH} \longrightarrow 2 \mathrm{H}_{2} \mathrm{O}+\mathrm{GSSG}$

If the generation of $\mathrm{OH}^{\bullet}$ is not controlled adequately because the dietary supply of these minerals is insufficient, then $\mathrm{OH}^{\bullet}$ may attack the unsaturated lipids of biological membranes, thus:

$\mathrm{LH}+\mathrm{OH}^{\bullet} \longrightarrow \mathrm{L}^{\bullet}+\mathrm{H}_{2} \mathrm{O}$

$\mathrm{L}^{\bullet}+\mathrm{O}_{2} \longrightarrow \mathrm{LOO}^{\bullet}$

The lipid peroxyl radical generated in this way may attack a further unsatured phospholipid adjacent to it in the membrane to initiate a chain reaction:

$\mathrm{LOO}^{\bullet}+\mathrm{LH} \longrightarrow \mathrm{LOOH}+\mathrm{L}^{\bullet}$

The generation of such chain reactions is however prevented efficiently when the intracellular concentration of vitamin $\mathrm{E}$ is adequate:

$\mathrm{LOO}+\mathrm{EH} \longrightarrow \mathrm{LOOH}+\mathrm{E}$

This chain-breaking function of vitamin $E$ leads to the formation of the vitamin $E$ radical $E$ : The precise mechanism for the regeneration of vitamin $E$ from its radical is not know, but it is believed to involve both glutathione and ascorbic acid (vitamin $\mathrm{C}$ ); a likely reaction sequence is:

$\mathrm{E} \cdot+$ Ascorbate $\longrightarrow \mathrm{EH}+$ Dehydroascorbate

Dehydroascorbate + GSH $\longrightarrow$ Ascorbate + GSSG

$\mathrm{GSSG}+\mathrm{NADPH}+\mathrm{H}^{+} \longrightarrow 2 \mathrm{GSH}+\mathrm{NADP}^{+}$

The biological role of vitamins $\mathrm{E}$ and $\mathrm{C}$ are thus intimately interlinked. 
The lipid hydroperoxide formed by either of the above reactions is not a 'safe' end-product because a haem iron-catalyzed reaction may cause generation of both peroxyl and alkoxyl radicals from it. This eventuality is preventive because glutathione peroxidase can catalyze the further reduction of the lipid hydroperoxide to hydroxy acid:

$\mathrm{LOOH}+2 \mathrm{GSH} \longrightarrow \mathrm{LOH}+\mathrm{H}_{2} \mathrm{O}+\mathrm{GSSG}$

Thus the antioxidant protective mechanism may be seen to be manifested at three levels; the enzymic mechanism, involving trace minerals, that removes the initiating species; the concerted action of vitamin $E$ and vitamin $C$ in breaking free radical-initiated chain reactions; and the further intervention of selenium-glutathione peroxidase in removing lipid hydroperoxides.

There may be other antioxidant mechanisms that form part of the protective mechanism. The work of Balasubramanian, in collaboration with the present author, has demonstrated that free monounsaturated fatty acids can, in certain circumstances, act as intracellular antioxidants. Thus, it was shown [5] that the resistance of gastro-intestinal mucosa to both non-enzymic and enzymic lipid peroxidation was due to the presence in the mucosa of quite large amounts of a lipid-soluble factor which differed significantly from tocopherol in its properties. Subsequent work [6] demonstrated that the inhibitory factor was a mixture of free fatty acids in which oleic and palmitoleic acids were the antioxidants.

The antioxidant ability of these fatty acids was shown [7] to occur only in systems in which the peroxidation was driven by metal ions and it was concluded that the inhibitory effect of the monoenoic fatty acids was due to iron (and perhaps copper) chelation by the fatty acid.

\section{REFERENCES}

[1] Diplock, A.T. (1991). Antioxidant nutrients and disease prevention: an overview. Amer. J. Clin. Nutr. 53, 189S-193S.

[2] Steinberg, D., Parthasarathy, S., Carew, T. E., and Witztum, J. L. (1989). Beyond cholesterol: modifications of low-density lipoproteins that increase its atherosgenicity. N. Engl. Med. 320, 915-924.

[3] Weisberger, J. H. (1991). Nutritional approach to cancer prevention with emphasis on vitamins, antioxidants and carotenoids. Amer. J. Clin. Nutr. 53, 226S-237S.

[4] Varma, S.D.. (1991). Scientific basis for medical therapy of cateracts by antioxidants. Amer. J. Clin. Nutr. 53, 335S-345S .

[5] Balasubramanian, K.A., Manohar, M., and Mathan, V. I. (1988) An unidentified inhibitor of lipid peroxidation in intestinal mucosa. Biochim. Biophys. Acta. 962, 51-58.

[6] Diplock, A.T., Balasubramanian, K.A., Manohar, M., Mathan, V.I., (1988). Purification and chemical characterisation of the inhibitor of lipid peroxidation in intestinal mucosa. Biochim. Biophys. Acta., 962, $42-50$.

[7] Balasubramanian, K.A., Nalini, S., Cheeseman, K.H., and Slater T.F. (1989). Nonesterified fatty acids inhibit iron-dependent lipid peroxidation. Biochim. Biophys. Acta. 1003, 232-237 